\title{
NONSTEADY STABILITY OF THE FLOW AROUND THE CIRCLE IN THE FÖPPL MODEL
}

BY

\author{
DANIELA TORDELLA
}

Politecnico di Torino, Torino, Italy

\begin{abstract}
The Föppl model for the incompressible flow about the circle is considered. The major feature of this model is the understanding of the convective mechanism that gives rise to the onset of the instability in the flow past the circle. Through this model the real flow is approximated by means of a potential field with two singular points, counter-rotating vortices, placed symmetrically behind the circle. This field configuration is topologically analogous to the actual steady field for Reynolds numbers below the critical value corresponding to the onset of the first instability. The intrinsic instability of the Föppl field to small perturbations may be described by a second-order linear dynamical system.

In this paper the instability behaviour of the Föppl field during transient motions is studied. It is shown that a reduction of the instability growth rate may be induced by a positive acceleration of the asymptotic stream or by a pulsating asymptotic stream. In this last case the effect is directly displayed by a reduction of the temporal growth rate of the perturbation with respect to that belonging to the steady state. In the first case the reductive effect is appreciated through a modified form of Shen's criterion. The relevance of these results to experimental observations of the real flow is discussed.
\end{abstract}

1. Introduction. Föppl's model has a historical interest: it was the first attempt to analytically justify the intrinsic instability of the incompressible flow about the circle that develops in real fluids when the critical value of the Reynolds number is exceeded [1].

For Reynolds numbers $(R e)$ greater than about 4 the flow pattern is characterized by the fact that the streamlines near the circle depart from it before reaching the rear point of symmetry, and therefore a continuously circulating bubble of fluid shows up behind the circle. Within the bubble the structure of the field is that of two standing eddies adherent to the back of the circle. As $R e$ is increased above the critical value, the flow in this region becomes unsteady, even if the imposed conditions are stationary.

Received March 15, 1993.

1991 Mathematics Subject Classification. Primary 76E99, 76C05. 
Föppl envisaged a field configuration, similar to the one corresponding to Reynolds numbers below the onset of instability, by employing the potential field-that describes the irrotational flow due to a circle of unit radius in an otherwise uniform stream-to which he superimposed, behind the circle, the field created by two distinct singular points. These points, where one must imagine finding two discrete vortices (as a consequence, inside the circle there will be placed two discrete image vortices), are symmetrically placed with respect to the direction of the asymptotic velocity. In doing so he simplified the problem drastically so as to offer an analytical representation of the flow itself.

Being devoid of control parameter the Föppl model is unable to predict the onset of instability. As a result, it shows that the field configuration with two steady vortices placed behind the circle is always unstable, while in the real viscous flow it is so only for $\operatorname{Re}>\operatorname{Re}_{c r}\left(\operatorname{Re}_{c r} \sim 40\right)$.

The salient point about this model is the picking out of the instability, that it describes as the breaking of the equilibrium due to a convective mechanism. Föppl shows that in the plane of the motion a branched curve exists, the locus of the couples of points where the vortices' centers may be found in equilibrium. These positions of equilibrium are unstable to the antisymmetric components of small perturbations, a fact that is of relevance because, in the real problem, the onset of instability is always associated with the breaking out of the flow symmetry. Not at random one must artificially symmetrize the flow to experience a stable supercritical flow around the cylinder, in the configuration with two attached standing and symmetric vortices behind the obstacle $[2,3]$.

This work is based on the hypothesis that the Föppl model describes with faith the outbursting convective mechanism of the first instability of the flow field past the circle. In it the influence of unsteady asymptotic velocity on the instability of the flow is studied in two cases: (a) in the case of uniformly accelerated or decelerated asymptotic motion and (b) in the case of harmonic oscillating asymptotic motion. Small displacements about the equilibrium position of the centers of the two vortices are introduced and their temporal growth is considered in these new conditions.

This problem may be reduced to the study of a linear second-order dynamical system, that in the particular case of a harmonic asymptotic oscillating motion is described by a Hill-Mathieu equation. The growth factors of the perturbations are analytically determined by means of the approximated methods of Liouville-Green (Case a) and Nocilla [4] (Case b). In this last case the characteristic coefficients are also computed by means of the Floquet theory, which has been applied after having numerically determined two principal, linearly independent, integrals of the motion.

By applying the stability criterion suggested by Shen [5] for unsteady flows, the perturbation growth rate, in the case of a uniform accelerated or decelerated asymptotic motion, has been studied and compared to the one given by the corresponding dynamical system with constant coefficients. When the asymptotic stream is uniformly accelerated or decelerated, the meaning of a greater or smaller growth rate of a disturbance is rather obscure. In order to evaluate the relative importance of the perturbation, we followed a slightly modified version of Shen's criterion by comput- 
ing the evolution in time of the ratio of the kinetic energy of the disturbance to that of the asymptotic flow. This way we obtained a sort of reductive effect in the case of accelerated motion and the opposite in the case of decelerated motion.

In the case of an oscillating asymptotic velocity it will be shown that the growth rate of small perturbations is always lower than that of the stationary case.

2. The Föppl model. Consider the two-dimensional motion of an incompressible inviscid and uniform stream flowing around the circle. The Föppl assumption about this flow is that the motion is mainly characterized by the presence of two discrete symmetric and counter-rotating vortices placed downstream of the circle; see Fig. 1. The model proposed by Föppl, which concentrates all the vortical region of the circle wake in two points, simplifies drastically the actual motions in such a way as to allow an analytic representation of the flow field.

The field is thus composed of a uniform stream $U$ in which the following singularities are placed: a dipole that represents the modification of the uniform stream due to the presence of the circle and two couples of vortices of intensity $C$ of which the inner is the image of the external one with respect to the circle boundary; see Fig. 1. The dipole strength is $G=2 \pi U$.

Föppl demonstrated that the two counter-rotating discrete wake vortices remain at fixed positions, i.e., they have no induced velocity, if their centers are placed on two symmetric points belonging to the branched curves:

$$
\pm 2 \eta=r-\frac{1}{r}
$$

where $\xi$ and $\eta$ are Cartesian coordinates of a system of reference placed in the center of the circle (the $\xi$-axis lies in the $U$ direction) and $r=\sqrt{\xi^{2}+\eta^{2}}$; see again Fig. 1. The vortices intensity $C$ will depend both on the undisturbed stream and on

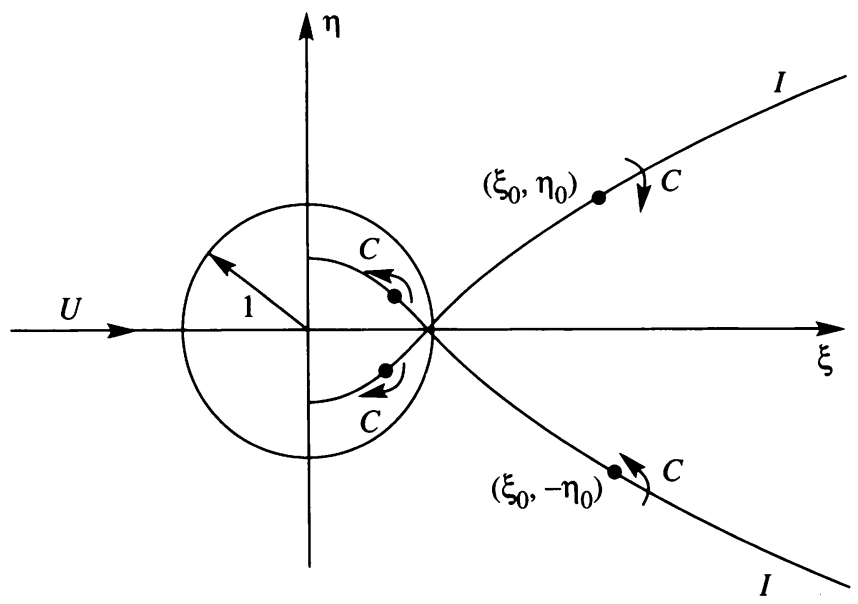

Fig. 1. Flow configuration 
the distance from the circle center:

$$
C=2 U \eta\left(r-\frac{1}{r^{4}}\right) \text {. }
$$

Analyzing the influence of a typical small (infinitesimal) perturbation on this flow configuration, Föppl found that the equilibrium points, i.e., all the couples of points $\left(\xi_{0}, \eta_{0}\right)$ and $\left(\xi_{0},-\eta_{0}\right)$, loci of the vortices' centers and belonging to the curves (1), are not stable. Any general perturbation of the vortices' center position might be decomposed into two classes of displacements, symmetrical and antisymmetrical with respect to the $\xi$-axis. Let us call $\alpha$ the displacement along the $\xi$-axis and $\beta$ the one along the $\eta$-axis; see Fig. 2. If $\alpha$ and $\beta$ are infinitesimally small with respect to the actual vortices' coordinates, $\left(\xi_{0}, \eta_{0}\right)$ and $\left(\xi_{0},-\eta_{0}\right)$, i.e.,

$$
\frac{\alpha}{\xi_{0}}, \frac{\beta}{\eta_{0}} \ll 1,
$$

it is possible to deduce from the complex potential of the field

$$
W=\Phi+i \Psi=U\left(\zeta+\frac{1}{\zeta}\right)+i C \ln \frac{\left(\zeta-\zeta_{0}\right)\left(\zeta+\frac{1}{\zeta_{0}}\right)}{\left(\zeta-\overline{\zeta_{0}}\right)\left(\zeta+\frac{1}{\zeta_{0}}\right)}
$$

the equations that describe the temporal evolution of these displacements (Föppl 1913). $\Phi$ and $\Psi$ are the potential and stream functions, while $\zeta=\xi+i \eta$ and $\bar{\zeta}=\xi-i \bar{\eta}$. By means of the kinematic relations:

$$
\begin{aligned}
& u_{0}=\frac{d \xi}{d t}=\frac{d\left(\xi_{0}+\alpha\right)}{d t}=\frac{d \alpha}{d t}=u_{0}\left(\xi_{0}, \eta_{0} ; C, U\right), \\
& v_{0}=\frac{d \eta}{d t}=\frac{d\left(\eta_{0}+\beta\right)}{d t}=\frac{d \beta}{d t}=v_{0}\left(\xi_{0}, \eta_{0} ; C, U\right),
\end{aligned}
$$

since on an equilibrium point we have $\frac{d \xi_{0}}{d t}=\frac{d \eta_{0}}{d t} \equiv 0$, Föppl obtained the following

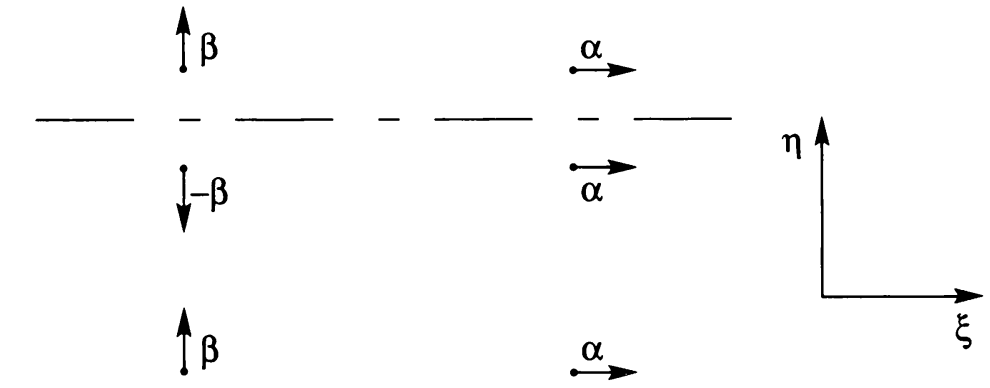

i)

ii)

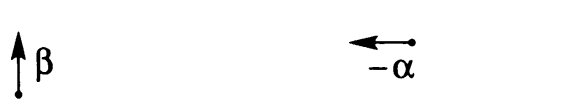

FIG. 2. Displacements system of the couple of wake vortices with centers at $\left(\xi_{0}, \eta_{0}\right)$ and $\left(\xi_{0},-\eta_{0}\right):$ i) symmetrical set of displacements, ii) antisymmetrical set of displacements. 
second-order differential systems:

$$
\begin{gathered}
\frac{d^{2} \alpha}{d t^{2}}+a U \frac{d \alpha}{d t}+b U^{2} \alpha=0 \quad \text { (for symm. displacements), } \\
\frac{d^{2} \alpha}{d t^{2}}+c U^{2} \alpha=0 \quad \text { (for antisymm. displacements), }
\end{gathered}
$$

where the coefficients $a, b, c$ are somewhat complicated, time independent, real functions of the spatial positions $\left(\xi_{0}, \eta_{0}\right)$ of the wake vortices.

Föppl's analysis showed that the system response to an arbitrary small perturbation amplifies the antisymmetrical displacements and damps the symmetrical ones. Since the more general perturbation contains always an antisymmetrical component, a global situation of instability takes place.

This result was used to analytically justify the first experimental observations done at the time [6] about the vortex shedding instability. Of course, this very synthetic description of the flow about the circle disregards any influence of the viscous diffusion. Anyway, the intrinsic instability of this flow field is clearly pointed out.

3. Unsteady motion. We now extend this analysis to the nonstationary situation, where the asymptotic uniform stream is time dependent. In such a case the flow field (solution of the Laplace equation) will adjust to the instantaneous asymptotic stream variation, since in this framework the propagation speed is infinite [7, 8]. Thus, Eqs. (4) and (5) will become nonautonomous with $U=U(t)$.

In the following we will be considering only the differential equation corresponding to the antisymmetric perturbation (5), because of its intrinsic instability. The analysis of Eq. (4) is devoid of interest. Owing to the term proportional to the first derivative $d \alpha / d t$, it is formally structured as the equation of a second-order dynamical system with a damping element. The corresponding autonomous equation is always stable, and the solutions are bounded for $t \rightarrow \infty$, since $a U$ and $b U^{2}$ are both positive [1]. When unsteady motion is set up the coefficients $a$ and $b$ will not change their sign in the surrounding of $t=0$, if the velocity variations are small with respect to the steady value. Therefore, the relevant solution remains bounded.

Coming back to Eq. (5) we now consider the following situations, under the hypothesis of small perturbations; see Fig. 3 on p. 688:

i) uniformly accelerated/decelerated motion, $U-U_{0}=h t$ with $h \gtrless 0, t \in\left[0, t^{\prime}\right]$ such that $\left|\frac{U-U_{0}}{U_{0}}\right|=\frac{h t^{\prime}}{U_{0}} \ll 1$;

ii) pulsating asymptotic motion, $U-U_{0}=\Delta u \cos \omega t$ with $\left|\frac{\Delta u}{U_{0}}\right| \ll 1$.

3.1. Flow with constant acceleration. In this situation the asymptotic velocity is $U(t)=U_{0}+h t$. The differential equation describing the temporal evolution of the vortices' center displacement is

$$
\frac{d^{2} \alpha}{d t^{2}}+c\left(U_{0}+h t\right)^{2} \alpha=0,
$$


i)

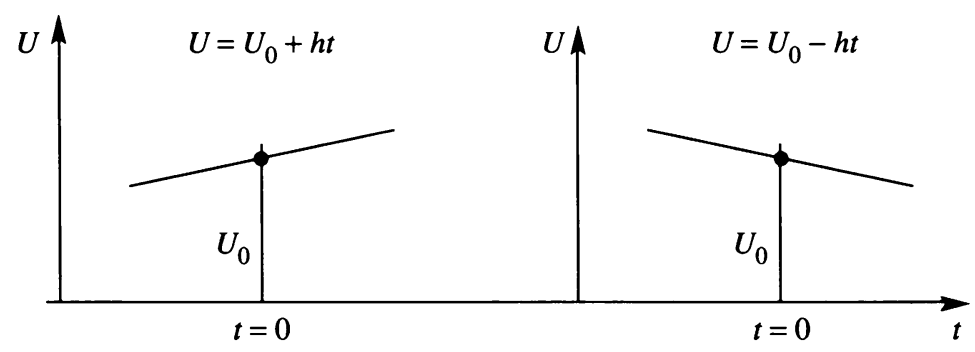

ii)

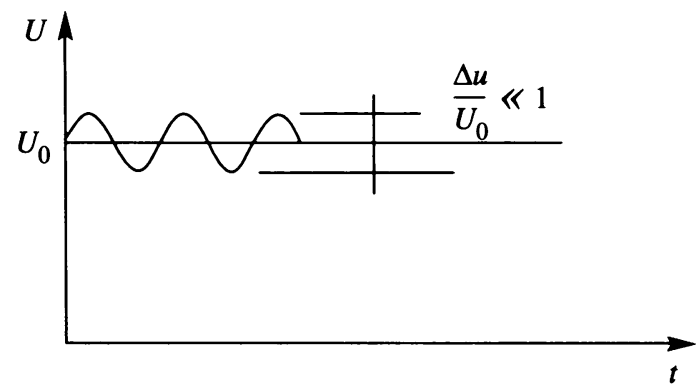

FIG. 3. i) Uniform accelerated/decelerated asymptotic motion.

ii) Asymptotic pulsating stream

where $U_{0}$ is the asymptotic speed at $t=0$ and $h$ is the acceleration. The coefficient $c$ (see Sec. 2) is a function of the spatial positions of the wake vortices and is always negative on the equilibrium curves $I-I$ and on their neighbourhoods; see again Fig. 1.

It is convenient to make the transformation $t / t^{*}=\tau$ where $t^{* 2}=-1 /\left(c U_{0}^{2}\right)$. In this way Eq. 6 becomes

$$
\frac{d^{2} \alpha}{d \tau^{2}}-\left(1+\frac{h}{U_{0}} t^{*} \tau\right)^{2} \alpha=0
$$

Setting $\frac{h}{U_{0}} t^{*}=\frac{h}{U_{0}^{2}} \frac{1}{\sqrt{-c}}=\lambda$ we obtain

$$
\frac{d^{2} \alpha}{d \tau^{2}}-(1+\lambda \tau)^{2} \alpha=0
$$

To evaluate the instability characteristics of this system it is convenient to use the Shen criterion [5] for the stability of unsteady flows undergoing infinitesimal disturbances:

$$
\mho_{E}=\frac{1}{E} \frac{d E}{d t} \leq 0
$$

where $E$ is a function representing the ratio of the kinetic energy of the disturbance

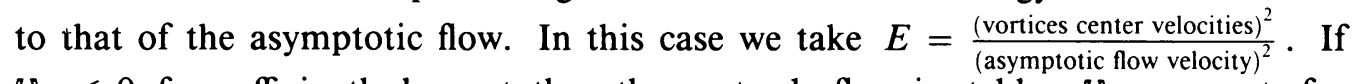
$\mho_{E} \leq 0$ for sufficiently large $t$ then the unsteady flow is stable. $\mho_{E}$ accounts for the relative evolution of the perturbation with respect to the asymptotic unsteady motion. Applying this criterion to system (7) it is straightforward to obtain the following result. By definition, $E=(d \alpha / d \tau)^{2} /(1+\lambda \tau)^{2}$ and, taking into account Eq. (7),

$$
\mho_{E}=\frac{1}{E} \frac{d E}{d \tau}=2\left[\frac{(1+\lambda \tau)^{2} \alpha}{d \alpha / d \tau}-\frac{\lambda}{(1+\lambda \tau)}\right],
$$


which at the initial instant $\tau=0$ gives

$$
\mho_{E_{\tau=0}}=2\left(\frac{\alpha_{0}}{\alpha_{0}^{\prime}}-\lambda\right),
$$

where $\alpha_{0}^{\prime}=(d \alpha / d \tau)_{t=0}$. Since the sign of $\alpha_{0} / \alpha_{0}^{\prime}$ depends on the initial conditions, the first term on the right-hand side of (10) does not have a predictable sign. On the other hand, we know for sure that the second term, i.e. $-2 \lambda$, will be

- stabilizing if $h>0 \quad(\lambda>0)$,

- unstabilizing if $h<0 \quad(\lambda<0)$.

Thus, whatever the absolute value is for the acceleration, of course within the limits allowed by condition i) in Sec. 3 (infinitesimal perturbation hypothesis), we obtain in the case of an accelerated motion an initial reduction of the speed of amplification of an infinitesimal small perturbation of the flow field relative to the steady motion condition, i.e., $h=0$.

Equation (7) can be reduced to the Bessel differential equation and has the general solution, see [9, pp. 437-442],

$$
\alpha(\tau)=T^{1 / 2} Z_{1 / 4}\left(i \frac{T^{2}}{2}\right)
$$

where $T=(1+\lambda \tau)$ and $Z_{1 / 4}$ is a linear combination with arbitrary coefficients of Hankel's functions.

At this point we would like to get a clue about the instability behaviour in the case of very slowly accelerated/decelerated flow, i.e., in the situation where this approximate model is physically more relevant, and in a short time interval starting from the initial instant $t_{0}$, so as not to overcome the validity boundaries of the linearization. The following alternative procedure is adopted. With the change of unknown function,

$$
\alpha=e^{\gamma(\tau)}, \quad z=d \gamma / d \tau,
$$

Eq. (7) is reduced to the Riccati equation

$$
d z / d \tau=(1+\lambda \tau)^{2}-z^{2}
$$

which for

$$
\left|\frac{h}{U_{0}} t^{*}\right|=\left|\frac{h}{U_{0}^{2}} \frac{1}{\sqrt{-c}}\right|=|\lambda| \ll 1
$$

has the following particular approximated solutions:

$$
z(\tau)= \pm(1+\lambda \tau)
$$

Thus, we have the corresponding approximated solution of Eq. (7), with the initial conditions:

$$
\begin{gathered}
\alpha(0)=\alpha_{0}, \quad \alpha^{\prime}(0)=\alpha_{0}^{\prime} \\
\alpha(\tau)=\frac{1}{2}\left[\left(\alpha_{0}+\alpha_{0}^{\prime}\right) \exp \left(\tau+\frac{1}{2} \lambda \tau^{2}\right)+\left(\alpha_{0}-\alpha_{0}^{\prime}\right) \exp \left(-\tau-\frac{1}{2} \lambda \tau^{2}\right)\right]
\end{gathered}
$$



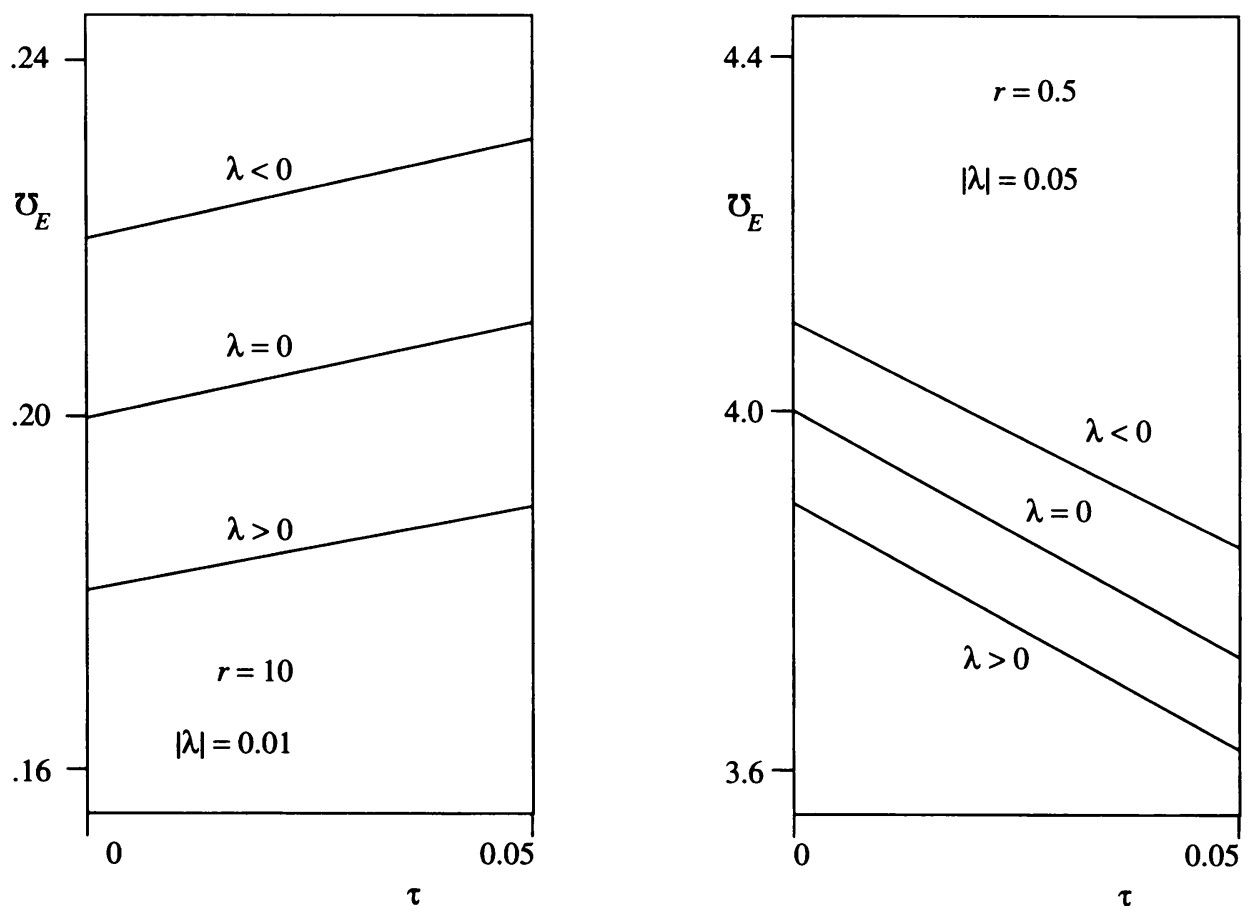

FIG. 4. Accelerated, decelerated, and steady motion with the same initial conditions: behaviour of $\mho_{E}$ around the initial instant

Inserting this value of $\alpha(\tau)$ into Eq. (9) we obtain the corresponding approximated value of $\mho_{E}$ :

$$
\mho_{E}=2(1+\lambda \tau) \frac{1+\frac{1-r}{1+r} \exp \left(-2 \tau-\lambda \tau^{2}\right)}{1-\frac{1-r}{1+r} \exp \left(-2 \tau-\lambda \tau^{2}\right)}-\frac{2 \lambda}{1+\lambda \tau}
$$

with $r=\alpha_{0}^{\prime} / \alpha_{0}$.

In Fig. 4 the behaviour around the initial instant of the approximated $\mho_{E}$ is plotted for accelerated, decelerated, and steady motion with the same initial conditions. Note that $\mho_{E}$ is not dependent on $\alpha_{0}$ and $\alpha_{0}^{\prime}$ separately but only on their ratio.

3.2. Asymptotic harmonic oscillations. Let us now consider the flow instability when $U(t)=U_{0}+\Delta u \cos \omega t$. Equation (5) then becomes a Hill equation:

$$
\frac{d^{2} \alpha}{d t^{2}}+c U_{0}^{2}\left(1+2 \frac{\Delta u}{U_{0}} \cos \omega t+\frac{\Delta u^{2}}{U_{0}^{2}} \cos ^{2} \omega t\right) \alpha=0 .
$$

Setting $\Delta u / U_{0}=\varepsilon$ and assuming $\varepsilon \ll 1$, we have

$$
U^{2}(t) \simeq U_{0}^{2}\left(1+2 \frac{\Delta u}{U_{0}} \cos \omega t\right)=U_{0}^{2}(1+2 \varepsilon \cos \omega t) .
$$

Thus, Eq. (12) reduces to the Mathieu equation:

$$
\frac{d^{2} \alpha}{d t^{2}}+c U_{0}^{2}(1+2 \varepsilon \cos \omega t) \alpha=0
$$


Furthermore, introducing the characteristic exponent of the steady problem

$$
\gamma_{s}^{2}=-c U_{0}^{2}
$$

we have

and, finally taking $\tau=\omega t$,

$$
\frac{d^{2} \alpha}{d t^{2}}-\gamma_{s}^{2}(1+2 \varepsilon \cos \omega t) \alpha=0
$$

$$
\frac{d^{2} \alpha}{d \tau^{2}}+(\lambda+\mu \cos \tau) \alpha=0
$$

with

$$
\lambda=-\frac{\gamma_{s}^{2}}{\omega^{2}} \quad \text { and } \quad \mu=-2 \frac{\gamma_{s}^{2}}{\omega^{2}} \varepsilon
$$

The assumption $\varepsilon \ll 1$ implies also $\mu \ll 1$. In this case it is possible to obtain the general integral by means of the approximate method of Nocilla [4], as in the following:

$$
\alpha(\tau) \simeq \exp ^{\gamma \tau}\left(1+A \exp ^{i \tau}+B \exp ^{-i \tau}\right)=\exp ^{\gamma \tau} \varphi(\tau)
$$

with

$$
\begin{gathered}
A=\frac{\mu}{2} \frac{1}{1+\beta-2 i \gamma}, \quad B=\frac{\mu}{2} \frac{1}{1+\beta+2 i \gamma}, \\
\beta=-\left(\lambda+\gamma^{2}\right), \\
\frac{\gamma^{2}}{\omega^{2}} \simeq-\frac{1}{4}\left(1-\sqrt{(1-4 \lambda)^{2}-4 \mu^{2}}\right) .
\end{gathered}
$$

Going back to the previous notation we have

$$
\gamma^{2} \simeq-\frac{\omega^{2}}{4}+\sqrt{\left(\frac{\omega^{2}}{4}+\gamma_{s}^{2}\right)^{2}-\gamma_{s}^{4} \varepsilon^{2}} .
$$

The value of $\gamma$ is always lower than $\gamma_{s}$, reaching $\gamma_{s}$ for $\varepsilon \rightarrow 0$ as it must be because in this case the asymptotic stream is no longer pulsating; see Fig. 5 on p. 692. In the surroundings of $\varepsilon=0, \omega=\omega^{*}$-where $\omega^{*}$ indicates any value of the pulsation of the asymptotic velocity large enough not to invalidate the condition $\mu \ll 1$, see Eq. (14) - the variation of $\gamma^{2}$ is more sensitive to the variation of $\varepsilon^{2}$ than to that of $\omega^{2}$ as can easily be shown by computing the first-order differential of $\gamma^{2}$ as a function of $\varepsilon^{2}$ and $\omega^{2}$.

This approximate solution, very useful in pointing out the relationship among the various physical quantities, has been checked against the exact solution of the Hill equation (11) obtained with the classical Floquet theory [10]. The general solution $\alpha_{1,2}=e^{\gamma_{1.2}} \phi(\tau)$ of that Hill equation is calculated as a linear combination of two principal solutions, satisfying opportune initial conditions, computed in the present work by means of numerical Runge-Kutta integration. Comparing $\gamma_{1,2 \text { Hill }}$ to $\gamma_{1,2 \text { Mathieu }}$, see Fig. 5, we see that the agreement is very good up to a value of $\varepsilon \sim 0.1$. Above this threshold the solutions start to diverge: Eq. (13) ceases to be a good approximation of the flow system. In Fig. 5 for $\varepsilon>0.1$ the $\gamma$ values of the exact Mathieu solutions are plotted. 


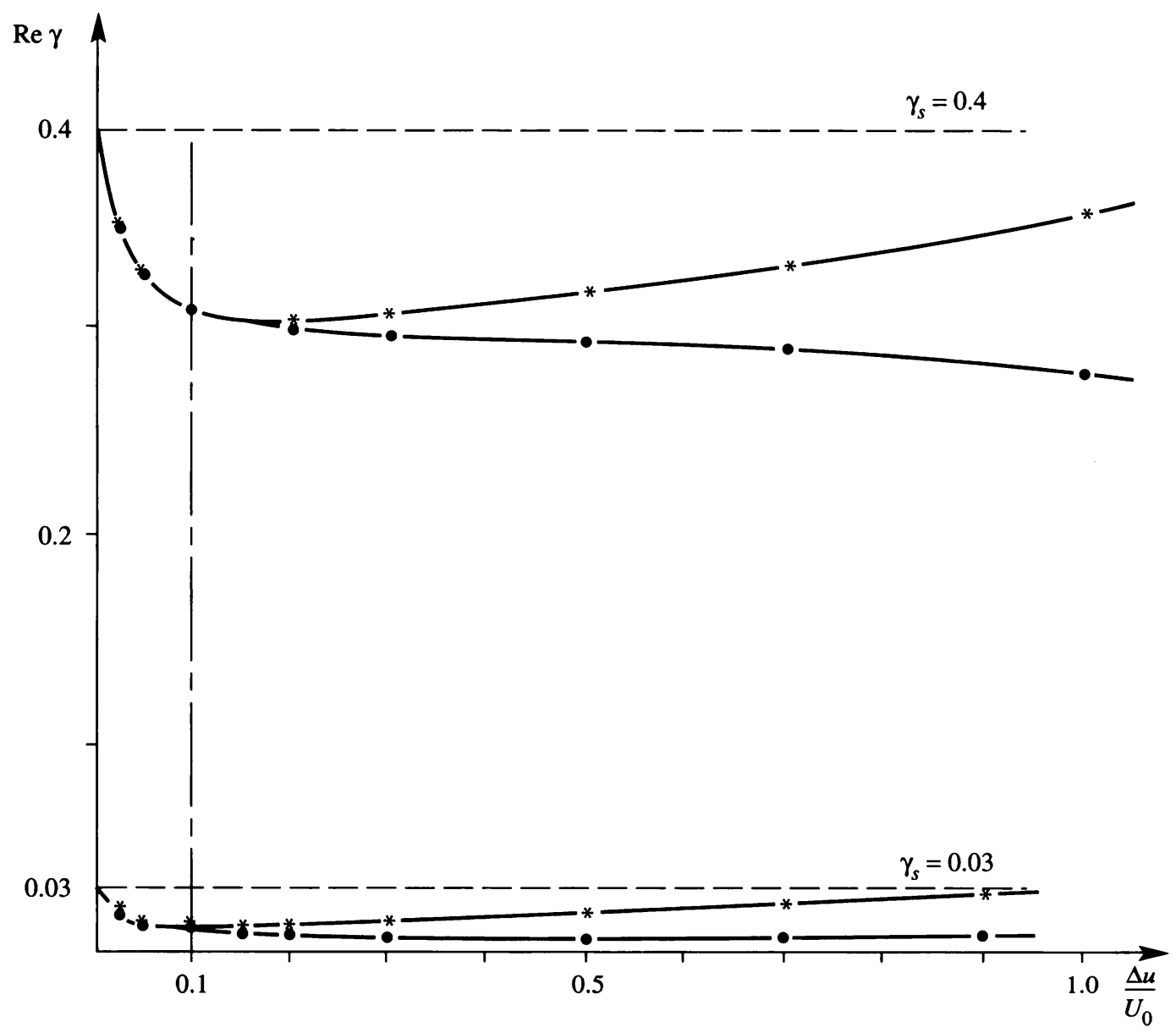

FIG. 5. Oscillation growing factor for the pulsating flow: - Mathieu equation $(\varepsilon \leq 0.1$ approximated Nocilla solution, $\varepsilon>0.1$ exact Mathieu solution). * Hill equation (Floquet theory). $\gamma_{\mathrm{s}}=$ stationary growing factor.

4. Discussion and conclusions. In this paper an investigation on the instability, during transient regimes, of the flow past the circle has been carried out, working in the frame of Föppl's model.

The Föppl model, being an application of the potential flow theory, is rather a rough approximation of the subcritical flow past the circle. The effects of the molecular diffusivity pertaining to the real flow are neglected. Nevertheless, it presents two positive aspects: first, the detection of the intrinsic flow instability, through the amplification of perturbations breaking out the field symmetry; second, its extreme analytical simplicity. Overall, the model may be conceived as yielding a good understanding of the convective mechanism that gives rise to the instability genesis. It was this point of view that prompted the curiosity of analyzing its instability behaviour under unsteady asymptotic conditions.

By means of this study, it has been shown that a positive acceleration is actually having some sort of reductive effect on the flow instability as viewed in the framework of the Föppl model. This result was obtained by applying Shen's criterion for the 
stability of unsteady flows, a criterion that evidences the relative temporal variation of the perturbation with respect to the asymptotic unsteady flow variation.

During the study of the situation characterized by a pulsating upstream velocity, another interesting result came along: it consists of the fact that the growth rate of the small perturbation is lower than the one corresponding to the steady state. This result, obtained through the approximate theory by Nocilla [4], has been checked by implementing a numerical computation of the exact solutions given by Floquet theory. In Fig. 5 the characteristic behaviours of the approximate and exact solutions are drawn. The agreement is very good up to a ratio of the pulsation amplitude to the mean velocity of about 0.1 . Above this value the original equation no longer holds because the assumption of small perturbations, leading to a linear second-order equation, is no longer valid. Quite apart from this fact, the growth rate of the exact solution of the Mathieu equation would decrease towards zero and so the system would become stable; see the Ince-Struttshe stability map [11]. Instead, the growth rate of the solution of the Hill equation decreases, with an increasing amplitude, to a minimum, after which it starts to increase continuously to overcome at a certain point the value that corresponds to the unperturbed case. In fact, the Hill equation does not present any region of stability for negative values of the parameter $\lambda$.

The fact that, theoretically, the growth rate of small perturbations could be lowered by pulsating the asymptotic velocity might be related to some experimental results by Berger [12], Wehrmann [13], and very recently by Schumm et al. [14], about the reduction of the velocity oscillations in a Karman vortex street. For instance, at Reynolds numbers above but very close to the critical value, by means of cylinder oscillations of "low amplitude and frequency slightly less than twice the natural Von Karman frequency" Berger showed that the forcing amplitude can be used as a control parameter of the vortex street onset.

Also, it may be interesting to recall that in fluid dynamics another example exists of reduction of a primary two-dimensional flow instability, through the imposition of asymptotic oscillatory conditions. With reference to the Kelvin-Helmholtz instability one may note an analogy between the present result and the suppression of the growth of the thickness of a shear layer forced at its fundamental frequency (low amplitude fluctuations, $[15,16,17])$.

The idea of investigating the effect of nonsteady, accelerated or decelerated, asymptotic conditions on Föppl's model originated from some experimental results obtained in the wake flow past a cylinder towed with slowly variable velocity in a still fluid [18], so as to perform a slow transition through the critical value of the Reynolds number. The response curves obtained in such a way displayed unexpected lineaments, such as a sort of hysteresis or a bursting of the signal in the neighbourhood of the critical value, which are not amenable to a first-order differential equation like Landau's [see 7, pp. 96-98]:

$$
\frac{d A}{d t}=\sigma A-\frac{l}{2} A^{3}
$$

even if a small unintentional irregularity of the asymptotic stream is supposed. 
A second-order differential equation, like Föppl's equation with variable coefficients, may obviously show a larger wealth of different behaviours. The reductive effects we have discussed up to now may be considered in a loose sense to be in agreement with the apparent hysteresis of the experimental results, if we assume a linking between a reduction of the perturbation growth rate of the unstable potential flow around the circle and a delay in the transition from steady to unsteady states of the actual flow around the same obstacle, which is not a straightforward argument, but we think that it may be considered an interesting clue.

Acknowledgments. I am grateful to Prof. S. Nocilla for valuable suggestions and comments.

\section{REFERENCES}

[1] L. Föppl, Wirbelbewegung hinter einem Kreiszylinder, Munich Akad. Wiss., Sitzungsb. d. Math.-Phys. Kl. Jahrg., 1913

[2] A. S. Grove, F. H. Shair, E. E. Petersen, and A. Acrivos, An experimental investigation of the steady separated flow past a circular cylinder, J. Fluid Mech. 19, 60-80 (1964)

[3] J. M. Cimbala and K. T. Chen, The behaviour of a freely rotatable cylinder/splitter plate body at post-critical Reynolds numbers, Bull. Amer. Phys. Soc. 37, 1772 (1992)

[4] S. Nocilla, Problemi insoliti di autovalori in meccanica non lineare, Rend. Mat. (4), Vol. 10, Serie VI, 1977

[5] S. F. Shen, Some considerations of the laminar stability of incompressible time dependent basic flows, J. Aerospace Sci. 28, 397-417 (1961)

[6] T. Von Karman and H. Rubach, Phys. Zeitschr., Jahrg., S. 49, 1912

[7] L. D. Landau and E. M. Lifshitz, Fluid Mechanics, Pergamon Press, Oxford, 1987, p. 18

[8] D. J. Tritton, Physical Fluid-Dynamics, Clarendon Press, Oxford, 1988, pp. 115, 116

[9] E. Kamke, Differentialgleichungen, B. G. Teubner, Stuttgart, 1977, pp. 437-442

[10] E. T. Whittaker and G. N. Watson, A course of modern analysis, Cambridge University Press, 1950, pp. $412-413$

[11] H. Kauderer, Nichtlineare Mechanik, Springer-Verlag, New York, 1958, pp. 503-511

[12] E. Berger, Suppression of vortex shedding and turbulence behind oscillating cylinders, Phys. Fluids 10, 191-193 (1967)

[13] O. H. Wehrmann, Reduction of velocity fluctuations in a Karman vortex street by a vibrating cylinder, Phys. Fluids 8, 547-761 (1965)

[14] M. Shumm, E. Berger, and P. A. Monkewitz, Self-excited oscillations in the wake of two-dimensional bluff bodies and their control, submitted for publication, 1992

[15] D. Oster and I. Wygnanski, The forced mixing layer between parallel streams, J. Fluid Mech. 123, $91-130(1982)$

[16] C. H. Ho and P. Huerre, Perturbed free shear layer, Annual Rev. Fluid Mech. 16, 365-424 (1986)

[17] D. Tordella and W. H. Christiansen, Spectral observation in a forced mixing layer, Vol. 27, 1989, pp. 1741-1743

[18] D. Tordella and C. Cancelli, First instabilities in the wake past a circular cylinder. Comparison of transient regimes with Landau's model, Meccanica 26, 75-83 (1991) 